who engage in age disparate sex compared to those who do not engage in age disparate sex.

Methods The data for this study comes from the third National HIV communication Survey (NCS) of South Africa conducted in 2012. The sample consists of 4065 randomly selected men between the ages of 16 and 55 years. Respondents were interviewed by means of a structured questionnaire. Men were classified according to the types of sexual partnerships they had engaged in during the previous 12 months. Multiple logistic regression models were used to assess the association between SES and sexual partnership type, controlling for potential confounders.

Results Sixty percent of men had been involved in only age similar partnerships, $31 \%$ in at least one age disparate partnership (partner is 5 to 9 years younger) and $10 \%$ in relationships where the female partner is 9 years or more years younger. The results indicate that household wealth is not significantly related to the likelihood of men engaging in age disparate sex or intergenerational sex. Interestingly, experiencing deprivation in informal urban areas increases men's likelihood of engaging in age disparate sex (AOR: 1.3, p<0.05).

Conclusion The results reveal that it is poorer men in informal urban settlements who are engaging in age disparate sex. Literature suggests that young women primarily engage in transactional sex with older men to support their basic needs. However, these data do not support this hypothesis and future research must identify the full range of factors fueling age disparate relationships.

\section{P4.44 PRIMARY HEALTHCARE WORKERS' PERCEPTIONS REGARDING CALLING SEXUAL PARTNERS OF PREGNANT WOMEN WITH SYPHILIS FOR TREATMENT}

Geraldo Bezerra da Silva Junior, Ana Fátima Braga Rocha, Kairle Santana Romualdo, Priscila de Sousa Xavier, Maria Alix Leite Araújo. University of Fortaleza, Fortaleza - CE, Brazil

\subsection{6/sextrans-2017-053264.541}

Introduction Due to low treatment coverage of sexual partners of pregnant women with syphilis in Brazil, this study aims to investigate the perceptions of primary healthcare workers about the calling of these partners for treatment.

Methods This is a descriptive study with qualitative designs conducted in six primary healthcare facilities including those with the highest numbers of pregnant women with syphilis in the city of Fortaleza, Ceará, Brazil. A total of 20 healthcare workers were included (seven physicians and thirteen nurses) and six healthcare unit's coordinators. The study was performed from June to September 2016 through semi-structured interviews, and content analysis was done.

Results According to interviewed healthcare workers, the calling and treatment of sexual partners of pregnant women with syphilis are complex and challenges activities, especially when they are not from the healthcare unit area or are illicit drug users. They pointed chauvinism as one of the main barriers and gender issues that causes resistance to go to the healthcare units for treatment. Lack of symptoms also makes partners believe they have no infection. There are also marital problems caused by the infection's diagnosis. The healthcare workers said not to feel able to these types of demands and require training. They believe that higher disclosures in different medias about syphilis and its consequences and "men prenatal care" would be important strategies to improve syphilis treatment coverage.

Conclusion Physicians and nurses in the primary care, as well as health units' coordinators, have several difficulties for calling and treating sexual partners of pregnant women with syphilis and do not feel able enough to face this problem.

\section{P4.45 INDIVIDUAL AND PARTNERSHIP FACTORS ASSOCIATED WITH ANTICIPATED VERSUS ACTUAL PARTNER NOTIFICATION FOLLOWING STI DIAGNOSIS AMONG MEN WHO HAVE SEX WITH MEN AND/OR WITH TRANSGENDER WOMEN IN LIMA, PERU}

${ }^{1}$ Hannan M Braun, ${ }^{2}$ Eddy R Segura, ${ }^{3}$ Javier R Lama, Jordan E Lake, ${ }^{3}$ Jessica Rios, ${ }^{3}$ Manuel V Villaran, ${ }^{3}$ Jorge Sanchez, ${ }^{5}$ Jesse L Clark. ${ }^{1}$ Doris Duke International Clinical Research Fellowship, UCLA Saphir Program, UCSF School of Medicine, San Francisco, CA, USA; ${ }^{2}$ UCLA Geffen School of Medicine, Lima - Peru; ${ }^{3}$ Asociacion Civil Impacta Salud y Educacion, Lima - Peru; ${ }^{4}$ McGovern Medical School at UThealth, Department of Internal Medicine, Division of Infectious Disease, Houston, TX, USA; ${ }^{5}$ UCLA Geffen School of Medicine, Department of Medicine, Division of Infectious Diseases, Los Angeles, CA, USA

\subsection{6/sextrans-2017-053264.542}

Introduction A detailed understanding of partner notification (PN) practices following STI diagnosis can improve PN strategies. Using data from 2 PN intervention trials in Lima, Peru, we assessed participant- and partner-level factors guiding partner-specific STI notification behaviour, including discordances between anticipated and actual notification.

Methods From 2012-14, newly STI-diagnosed (gonorrhoea, chlamydia, syphilis) men who have sex with men (MSM) and/ or with transgender women in Lima reported recent partners' characteristics, anticipated PN practices, and actual PN outcomes 14 day following STI diagnosis. In this sub-analysis of control-arm participants, GEE Poisson regression analyses assessed factors guiding PN outcomes.

Results Participants $(\mathrm{n}=150)$ predominantly identified as homosexual (70\%) and moderno (versatile sexual role, 55\%); $55 \%$ of partners $(n=402)$ were casual. $35 \%$ of partners were notified overall, though only $51 \%$ of anticipated PN occurred and $26 \%$ of notifications were unanticipated. $47 \%$ of participants did not notify any partners, while $24 \%$ notified all partners. PN was more frequent for main vs. casual (adjusted prevalence ratio $[\mathrm{aPR}] 0.55, \mathrm{p}<0.01$; adjusted for anticipated $\mathrm{PN})$ or commercial (aPR $0.29, \mathrm{p}<0.05)$ partners, with a trend toward notifying partners that used condoms (crude PR 1.30, $\mathrm{p}=0.09$ ). PN frequency did not differ by STI diagnosis. Anticipated PN predicted actual PN (aPR 1.60, p<0.01) imperfectly as $81(54 \%)$ participants' PN practices did not match anticipated behaviour. Successful notification despite anticipated silence (40 participants, 63 partners) was associated with stable partnerships and perceived community norms supporting PN. Failure of PN despite intent (43 participants, 73 partners) more frequently occurred when oral sex with the partner was exclusively reported, with a trend towards identifying the partner as activo (insertive role).

Conclusion Anticipated PN imperfectly reflects actual PN behaviour. Future interventions to improve notification should acknowledge the differing partnership contexts maintained by MSM. 


\section{P4.46 INTRODUCTION OF RAPID SYPHILIS AND HIV TESTING IN PRENATAL CARE IN COLOMBIA: QUALITATIVE ANALYSIS}

${ }^{1}$ Hernando G Gaitan Duarte, ${ }^{1}$ Maria Teresa Ochoa-Manjarres, ${ }^{2}$ Sidia Caicedo, ${ }^{3}$ Berta Gomez, ${ }^{4}$ Freddy Perez. ${ }^{1}$ Universidad Nacional de Colombia, Bogota D.C. - Colombia; ${ }^{2}$ Ministerio de Salud y La Protección Social, Bogota D.C. - Colombia; ${ }^{3}$ Pan American Health Organisation, Bogota D.C. - Colombia; ${ }^{4}$ Pan American Health Organisation, Washington, D.C, USA

\subsection{6/sextrans-2017-053264.543}

Introduction Interpret perceptions of Colombian health professionals concerning factors that obstruct and facilitate the introduction of rapid syphilis and HIV testing in prenatal care services.

Methods A qualitative study based on semi-structured interviews was carried out. A convenience sample was selected with 37 participants, who included health professionals involved in prenatal care services, programs for pregnant women, clinical laboratories, and directors of health care units or centres, as well as representatives from regional departments and the Ministry of Health.

Results Colombia does not do widespread screening with rapid syphilis and HIV tests in prenatal care. The professionals interviewed stated they did not have prior experience in the use of rapid tests-except for laboratory staff-or in the course of action in response to a positive result. The insurance system hinders access to timely diagnosis and treatment. Health authorities perceive a need to review existing standards, strengthen the first level of care, and promote comprehensive prenatal care starting with contracts between insurers and health service institutional providers. Participants recommended staff training and integration between health-policymaking and academic entities for updating training programs Conclusion The market approach and the characteristics of the Colombian health system constitute the main barriers to implementation of rapid testing as a strategy for elimination of mother-to-child transmission of syphilis and HIV. Measures identified include making changes in contracts between insurers and health service institutional providers, adapting the timing and duration of prenatal care procedures, and training physicians and nurses involved in prenatal care.

\section{P4.47 MODELLING THE IMPACT OF A HYPOTHETICAL VACCINE THAT PREVENTS HSV-2 BUT INCREASES SUSCEPTIBILITY TO HIV}

Ian Spicknall, Harrel Chesson. Centres for Disease Control and Prevention, Atlanta, GA, USA

\subsection{6/sextrans-2017-053264.544}

Introduction An HSV-2 vaccine could reduce the health and economic burden of HSV-2, including neonatal herpes. However, such a vaccine might increase susceptibility to HIV infection through immune cell recruitment to genital tissues. If so, HSV-2 vaccination may decrease HSV-2 incidence but also increase HIV incidence.

Methods We explored these tradeoffs in a mathematical model of HSV-2 transmission dynamics and vaccination that includes static HIV incidence using ordinary differential equations. We modelled scenarios resembling the United States in terms of HSV-2 and HIV transmission. We examined scenarios where HSV-2 vaccination either did or did not increase HIV susceptibility. We assumed that HSV-2 infection led to increased HIV susceptibility, but no other interactions between HSV-2 and HIV.

Results Assuming vaccine efficacy against infectiousness of 0.5 , vaccination may decrease HSV-2 incidence by $22 \%$. However, when vaccination increases HIV susceptibility, this benefit comes at the cost of increased HIV incidence: 1.6, 2.0, or $2.5 \%$ in populations with low, moderate, and high HIV exposure rates, respectively. When vaccination does not increase HIV susceptibility, HIV incidence decreased by 7.0, 6.1, and $4.9 \%$, respectively. The magnitude of these effects depend on additional factors such as the degree to which HSV-2 vaccination increases HIV susceptibility, HSV-2 vaccine coverage, and baseline HSV-2 prevalence.

Conclusion Our analysis shows how models can describe tradeoffs of public health interventions offering substantial benefits and possible harms. Models can be used to weigh the relative benefits and costs of a specific intervention, or to examine whether an intervention results in a net gain or net loss in quality adjusted life years. Our analysis illustrates the need for updated estimates of the quality-of-life impact of HSV-2 in different individuals in order to weigh the public health benefits of HSV-2 vaccination against potentially adverse outcomes. More research is needed to better describe the interaction between HSV-2 and HIV infection and transmission.

\section{P4.48 PERCEPTION OF SOCIAL SUPPORT BETWEEN PEOPLE AND PEOPLE WITH HIV}

Jacqueline Silveira de Quadros, Stela Maris de Mello Padoin,Cristiane Cardoso de Paula. Universidade Federal de Santa Maria, Santa Maria - RS, Brazil

\subsection{6/sextrans-2017-053264.545}

Introduction Women living with Human Immunodeficiency Virus (HIV) are faced with personal, family and social difficulties, which can lead to non-compliance with measures to prevent vertical HIV transmission. One strategy in coping with the disease is the social support that plays a role in mitigating the negative effects of stressful events related to infection. The objective was to evaluate if the social support perceived by pregnant women is different from that perceived by puerperal women living with HIV.

Method This is a cross-sectional survey carried out in the municipality of Santa Maria - RS, Brazil. We used an instrument composed of a characterisation questionnaire; Social Support Scale for People Living with HIV/AIDS. The data collection was from April to November 2014, with 78 pregnant and postpartum women. Descriptive statistics, Student's T or Mann-Whitney test and linear logistic regression were used for the analysis of the data. The level of significance was considered with values equal or less than $5 \%$.

Results It was observed that being a pregnant woman is a protection factor when compared to being puerperal, being the chance of protection equal to 8840 times (CI: 1,012-16,767) for general social support, and 6010 times (IC: 1,532-10,489) for support Social instrumental.

Conclusion Health professionals need to know and interact with the social support network of these women, in order to implement actions that help health care during this period of family reorganisation. In addition, it is necessary to expand humanised attention and strengthen public policies. 


\section{P4.49 ADOLESCENTS KNOWLEDGE AND AWARENESS CONCERNING HIVIAIDS AND FACTORS AFFECTING THEM IN DONYO SABUK, KENYA}

Jacquiline Nyambura Njeru. Mukunike CBO, Donyo Sabuk, Kenya

10.1136/sextrans-2017-053264.546

Introduction Adolescents are more vulnerable than adults on sexually transmitted diseases and HIV/AIDS. Among the adolescents, girls are more vulnerable to STDs including HIV/ AIDS. Their knowledge about STDs and HIV/AIDS is very poor. This study was conducted between February 2016 and September 2016 investigating adolescent's between 10-19 years knowledge about sexually transmitted diseases including HIV/AIDS, its mode of transmission and prevention methods.

Method A multistage cluster sampling technique was used to select the sample. Data on 300 upper primary school female adolescents and 300 high school female adolescents was analysed. The study found that a large proportion of upper primary school adolescents ages between10-14 had very little knowledge on sexually transmitted diseases and HIV. More than three quarter of high school adolescents ages 15-19 years had knowledge about HIV respectively. On an average, about one third of them had no knowledge on HIV in terms of modes of transmission and prevention.

Results The multivariate logistic regression analysis revealed that adolescent age, years of schooling and knowledge on STIs and STDs appeared to be important predictors of the awareness about HIV/AIDS.

Conclusion Useful and fruitful awareness campaigns to educate the upper primary female adolescents regarding the health consequences of STDs including HIV/AIDS and integrated approach is strongly suggested for creating knowledge and awareness to control the spread of HIV/AIDS among adolescents in Ndonyo Sabuk, Murang'a County, Kenya.

\section{P4.50 ASSOCIATIONS OF HIV TESTING WITH HIV STIGMA: IMPLICATIONS FOR FAITH BASED HIV TESTING AND TREATMENT}

${ }^{1}$ J.M Stewart, C Budhathoki,. ${ }^{2}$, D Bellinger,. ${ }^{3},{ }^{1}$ J.B Hamilton, . 'Johns Hopkins University School of Nursing, Department of Community Public Health, Baltimore, MD 21205, USA; ${ }^{2} J o h n s$ Hopkins University School of Nursing, Department of Acute and Chronic Care, Baltimore, MD 21205, USA; ${ }^{3}$ Johns Hopkins University School of Nursing, Baltimore, MD 21205, USA

\subsection{6/sextrans-2017-053264.547}

Introduction The promotion of HIV testing for African Americans is a key approach to reducing both the number of individuals that are unaware of their status as well as the transmission of HIV. African American churches can serve as alternative venues to promote HIV testing and linkage to care. However, HIV stigma serves as a barrier to the integration of HIV testing in churches. Results are mixed as to the levels of HIV stigma among African American churches.

Methods We surveyed the congregants of African American churches $(n=177)$ and compared participants from two types of churches, those with and without an HIV testing facility.
Results The HIV stigma score was not significantly different between congregants at churches with HIV testing and those without testing. However the participants from churches that did not offer HIV testing showed significantly different views on homosexuality, sexuality and drug abuse as barriers to HIV testing compared to those who attended churches that offered HIV testing $(\mathrm{p}<0.001)$.

Conclusion These results have implications for the important role that African American churches could have in not only offering HIV testing but reducing associated barriers.

\section{P4.51 ENGAGING YOUNG PEOPLE FROM MIGRANT AND REFUGEE BACKGROUNDS WITH SEXUAL AND REPRODUCTIVE HEALTH PROMOTION AND CARE IN SYDNEY, AUSTRALIA}

Jessica Botfield, Christy Newman, ZWI Anthony. UNSW Australia, Sydney, Australia

\subsection{6/sextrans-2017-053264.548}

Introduction Migrant and refugee young people are often underserved by mainstream sexual and reproductive healthcare $(\mathrm{SRH})$ in Australia and other high-income countries. Research is being undertaken to explore this, with particular emphasis on understanding the views and experiences of these young people regarding services for this aspect of health. In addition, we are exploring community, provider and policy perspectives on the complexities of engaging this diverse range of young people.

Methods A structured scoping review was undertaken to synthesise the contributions of research to understanding this topic and identify gaps in the literature. Semi-structured interviews were conducted with 23 key informants (health service providers, policymakers, academics, community advocates) to elicit their views on the complexities of, and best practice approaches to, engaging this group with SRH promotion and care. Semi-structured interviews with migrant and refugee young people are currently underway.

Results The scoping review identified two knowledge gaps: 1) perspectives of migrant and refugee young people regarding $\mathrm{SRH}$ health care, and 2) engagement of this group with SRH services. Key informant interviews revealed pivotal aspects of how professionals navigate this field: 1) appreciating the complexities of cultural diversity; 2) recognising structural barriers and disincentives to engagement; 3) normalising sexual health; 4) balancing 'youth-friendly' and 'culturally-competent' priorities; and 5) going beyond simple language translation to support open dialogue and meaningful engagement.

Conclusion As many high-income countries undergo demographic expansion and diversification due to migration, integrated and appropriate nation-wide frameworks for the design and delivery of SRH promotion and care is essential. The current phase of research seeks to document the views of migrant and refugee young people, aiming to capture both the synergies and divergences that are likely to be apparent between professional perspectives and the lived experience of this group of young people. 